\title{
Destination unknown
}

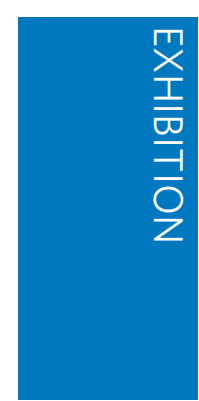

To many, space will always be the final frontier and galaxies far, far away. But science fiction, with its thousands of complex, distinct universes situated in futuristic worlds and dystopian regimes, is so much more. Popular culture's love affair with this genre rooted in science has stood the test of time, whichever way you travel through it, and it continues to resonate worldwide. As our own society has advanced, sci-fi has also evolved to explore deeper and more unique ideas. Part of the Barbican's 2017 Film in Focus programme, the new exhibition Into the Unknown, by Swiss historian and writer Patrick J. Gyger, delves into these worlds and celebrates our fascination from a new global perspective.

Our journey through science fiction begins with the four chapters featured in Into the Unknown: Extraordinary Voyages, Space Odysseys, Brave New Worlds and Final Frontiers, each of which introduces themes and ideas found throughout the wider experience.

Extraordinary Voyages, the first destination on this adventure, guides the visitor into the early works of sci-fi based on Earthly exploration and quests into our planet's unknown. A clear focus in this chapter is placed on classic European works, and Jules Verne is rightly at the forefront with his books on fantastical travels. Journeys are not the only thing covered here, though, with the massive reptiles of Godzilla, Dinotopia and Jurassic Park displayed too, as well as the art of the influential Ray Harryhausen.

It might be surprising to see Edgar Allan Poe, Arthur Conan Doyle and even Homer mentioned at this stage in an exhibition dedicated to sci-fi. However, starting chronologically, with what you might call proto-science fiction, provides comparison for what is to come.

The take-off into Space Odysseys, the largest section of the show, launches you into the most recognizable aspects of sci-fi. Iconic spacesuits, helmets and models appear from Alien, Star Wars and Star Trek, as well as recent major motion pictures such as Interstellar. The interactive NASA Mission Control set from The Martian continues this weaving of new and old and immerses the visitor in the film - sans botany. Astro Black by Australian art duo Soda_Jerk stands out as the first example of a new modern

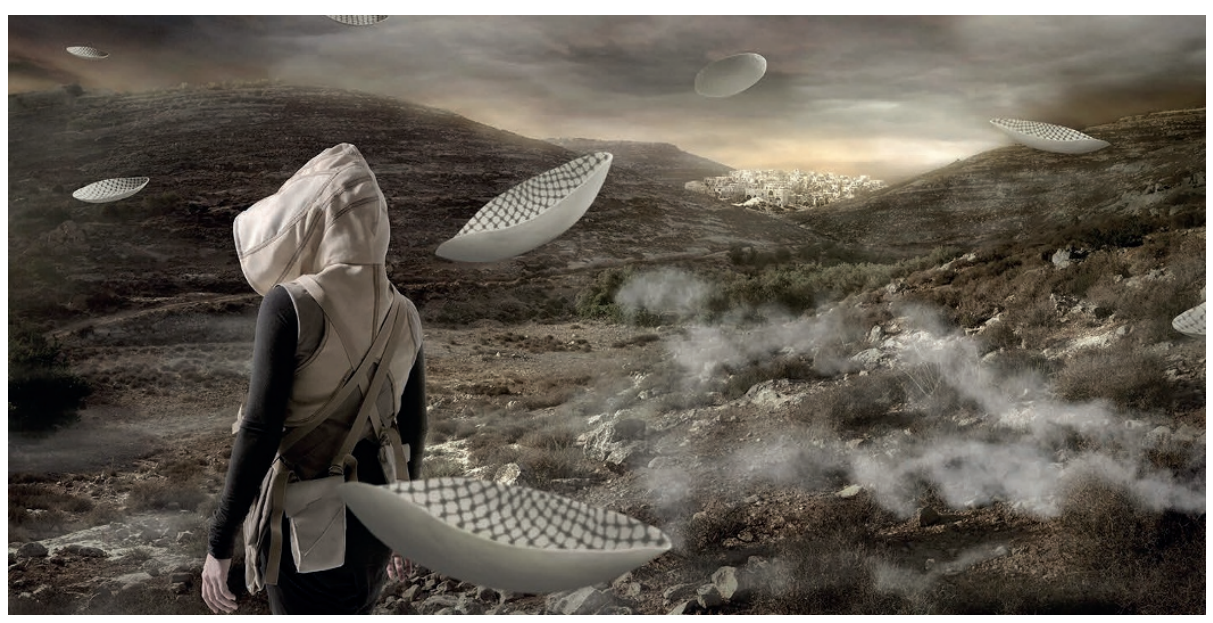

Image credit: Into the Unknown: A Journey Through Science Fiction, Film Still. In the Future They Ate from the Finest Porcelain, Larissa Sansour/Søren Lind, 2015. Courtesy of the artist and Sabrina Amrani.

perspective, with their use of video sampling and collage creating new associations between historical narratives.

After the expansive universes of the previous chapters, Brave New Worlds brings the visitor back to Earth in more ways than one. This section examines dystopian future cities and the downfall of civilizations, primarily through architecture - with drawings, plans, digital artwork and a selection of film clips. Pierre-Jean Giloux's featured video Metabolism \# Invisible Cities \# Part 1 set in Tokyo offers an interesting inspection of the role of structures in society. The artist's concept of alternative fluid-like buildings redeems this chapter, which comes across as comparatively narrow in scope.

Mind, body and the meaning of existence combine in the last chapter: Final Frontiers. Here the exhibition is brought to a close by looking ahead at the new horizons of technology. A selection of mid-twentieth century toy robots from Asia appear side by side with, and in stark contrast to, the lifelike android displays of Ex Machina and I, Robot. The progress of machines and their intelligence is further emphasized by the experimental short film Sunspring. Written entirely by an AI bot, Benjamin, the film uses neutral networks to challenge the unique nature of human creativity. In a similar vein, an auto-encoded version of Blade Runner reconstructed by artificial neural networks provides a glimpse into a future that won't, "like tears in rain", be lost in time.
Outside of the traditional exhibition is where Into the Unknown really flourishes, with its festival-style approach. By encouraging movement around the Barbican Centre you are rewarded for seeking out what else there is on offer. Music and video games can be enjoyed and the Kenyan short film Pumzi by Wanuri Kahiu can be seen before slipping into a dark studio to view Larissa Sansour and Søren Lind's Middle Eastern-focused In the Future They Ate from the Finest Porcelain (pictured). Delving down into the Pit you will find Conrad Shawcross's sculpture In Light of the Machine, a seemingly sentient mechanical robotic arm that casts light through slotted structures designed to explore the physics of light using technology.

Into the Unknown's global perspective on science fiction shines throughout the Barbican. The familiar, well-loved works and masterpieces situate and support the new art forms from around the world in perfect harmony. Classics lead into perhaps soonto-be fan favourites, and invite the visitor to consider new approaches to the genre. This sci-fi experience showcases the imagination of the genre and its evolution alongside science and technology.

\section{REVIEWED BY ADAM COX}

Into the Unknown: A Journey through Science Fiction opened on 3 June at the Barbican Centre in London and will run until 1 September 2017. 